\title{
La Unión de Universidades de América Latina y el Caribe y su papel en la defensa de la autonomía universitaria
}

\author{
ENRIQUE GRAUE WiECHERS
}

Rector de la Universidad Nacional Autónoma de México desde noviembre de 2015

\section{El movimiento autonómico universitario en América Latina: de la Reforma universitaria de Córdoba al Primer Congreso Universitario Latinoamericano}

La autonomía universitaria como la conocemos hoy es un fenómeno fundamentalmente latinoamericano que empezó a gestarse con el movimiento estudiantil de la Federación Universitaria de Córdoba, Argentina, en 1918.

La llamada Reforma de Córdoba fue un suceso histórico que supuso una reconfiguración radical en las relaciones entre las universidades, las sociedades y los estados latinoamericanos. En virtud de ella, los actores dueños del poder político y económico perdieron su influencia y control sobre las universidades, permitiendo a las clases medias urbanas acceder y participar en la toma de decisiones. Éstas irrumpieron en los recintos universitarios con la firme intención de cultivarse en las diferentes áreas del conocimiento y establecer un sistema de movilidad social basado en el mérito y no en la herencia. ${ }^{1}$

La lucha por la autonomía universitaria en Argentina fue un proceso intrínsecamente relacionado con los cambios políticos, sociales y económicos de la época. En el siglo XIX, con el ascenso del liberalismo político, que supuso el triunfo del republicanismo sobre las formas de gobierno autocráticas, la Universidad se deshizo en gran medida del control de la Iglesia ${ }^{2}$ y quedó supeditada al Estado en un acuerdo que entonces parecía beneficioso para ambas partes: la Universidad sería apoyada en su desarrollo en tanto proveyera de cuadros profesionales ${ }^{3}$ a los gobiernos liberales para modernizar al país.

En las dos últimas décadas del siglo XIX, bajo el gobierno de la llamada República Conservadora, Argentina experimentó un crecimiento económico, poblacional y cultural extraordinario con base en cuatro procesos mutuamente reafirmantes: la explotación de la tierra para la agroexportación, las inmigraciones europeas, la expansión de la red ferroviaria y la colonización de territorios. ${ }^{4}$ Sin embargo, este modelo pronto demostró su 
insuficiencia ante las dinámicas de concentración del poder político y económico, en una clase de oligarcas terratenientes y políticos corruptos que perdieron de vista el ascenso de la sociedad de masas y el surgimiento de clases sociales con nuevas expectativas.

A principios del siglo XX Argentina experimentó un cambio sociodemográfico fundamental a raíz de las olas migratorias provenientes del continente europeo, que engrosaron las clases medias y ayudaron a impulsar el desarrollo del comercio y la industria.

De acuerdo con Renate Marsiske (investigadora de la UNAM y experta en la historia de los movimientos estudiantiles latinoamericanos), las nuevas dinámicas sociales fueron la clave del movimiento estudiantil de Córdoba y de los que le sucedieron en otros países de América Latina. El desarrollo económico - sin precedentes- de la región empoderó a grupos de las clases medias urbanas con ambiciones políticas, los cuales, en varios contextos, lograron desafiar a las élites históricamente dominantes. ${ }^{5}$

Lejos de lo que se podría pensar, el proceso que llevó a la autonomía de la Universidad de Córdoba no fue impulsado por los sectores populares, sino por las segundas y terceras generaciones de migrantes, bien acomodados en la jerarquía social, que demandaron acceso a la educación superior en favor de sus gradualmente mayores aspiraciones políticas, culturales y económicas.

A principios del siglo xx era claro que la estructura interna de la universidad argentina, el contenido de sus enseñanzas y la forma de transmitir el conocimiento, no estaban a la altura de una sociedad cada vez más moderna. Las instituciones de educación superior eran dominadas por miembros de las oligarquías salientes y en algunos casos por fuerzas tradicionalistas relacionadas con la iglesia católica. Así, mientras la sociedad experimentaba cambios en su composición interna, la universidad seguía ensimismada en esquemas obsoletos.

Respecto a la docencia en Córdoba, y en otros recintos universitarios, se privilegiaba la memorización, el dogmatismo, la erudición sin sentido y materias humanísticas impregnadas aún del pensamiento positivista. Se producía poco conocimiento propio, lo cual reproducía los conocimientos generados en occidente y no creaba soluciones a los problemas locales específicos. Los egresados no recibían una educación suficiente o pertinente para hacer frente a los grandes problemas nacionales y las expectativas sociales de las clases medias. ${ }^{6}$

En ese sentido, el conflicto universitario fue producto de la gran discrepancia entre la estructura social cambiante y la estructura de la educación universitaria, el cual quedó claramente esbozada en el Manifiesto Liminar de los líderes reformistas cordobeses. ${ }^{7}$

Hombres de una república libre acabamos de romper la última cadena que en pleno siglo Xx nos ataba a la antigua dominación monárquica y monástica [...]. Las universidades han llegado a ser así el fiel reflejo de estas sociedades decadentes, que se empeñan en ofrecer el triste espectáculo de una inmovilidad senil. Los métodos docentes estaban viciados de un estrecho dogmatismo, contribuyendo a mantener a la Universidad apartada de la Ciencia y de las disciplinas modernas. Las lecciones ence- 
rradas en la repetición interminable de viejos textos, amparaban el espíritu de rutina y de sumisión [...].

La juventud ya no pide. Exige se le reconozca el derecho a pensar por su propia cuenta. Exige también que se le reconozca el derecho a exteriorizar ese pensamiento propio de los cuerpos universitarios por medio de sus representantes. Está cansada de soportar a los tiranos. Si ha sido capaz de realizar una revolución en las conciencias no puede desconocérsele la capacidad de intervenir en el gobierno de su propia casa. ${ }^{8}$

En contra del autoritarismo académico, las cátedras vitalicias, el acceso a la educación superior limitado por el elitismo, el dogma religioso y del saber a partir de los libros y las palabras antes que la práctica y los conceptos, los reformistas de Córdoba pidieron autonomía frente al Estado para poder materializar una serie de demandas concretas: concursos públicos de oposición para la provisión de cargos docentes, periodicidad de las cátedras para su renovación y actualización, docencia y asistencia libres, participación de los universitarios en el gobierno de la universidad, elección democrática de las autoridades, acceso incluyente de la población y planes de estudios actualizados con base en métodos modernos de enseñanza. ${ }^{9}$

Las reformas conseguidas durante ese momento histórico tuvieron tal impacto que el movimiento por la autonomía se difundió en muchas de las naciones latinoamericanas. En su manifiesto, los miembros de la Federación Universitaria de Córdoba vaticinaron una revolución, "una hora americana". Y así fue: los elementos ideológicos del movimiento estudiantil argentino reverberaron en todas las universidades de América Latina. Prueba de ello fueron los movimientos estudiantiles de la Universidad de San Marcos, en 1919; de la Universidad de la Habana, en 1922; de la Universidad Nacional de México, en 1929; y de la Universidad de San Carlos de Guatemala, en 1944.

Estos movimientos tenían en común el genuino interés de democratizar las decisiones de la vida universitaria para conectarse mejor con su entorno y con la sociedad que les había dado origen. Los jóvenes latinoamericanos releyeron y reinterpretaron los postulados de Córdoba para integrarlos en espíritu a sus sistemas legales y dar solución a los problemas propios de sus países, los cuales, si bien eran distintos a los enfrentados por los reformistas de 1918, suponían reivindicaciones similares. ${ }^{10}$ Fueron movimientos impulsados por la necesidad de cambiar las estructuras existentes, fortalecer la participación de profesores y estudiantes en los asuntos universitarios y colocar a la universidad como el principal centro generador de conocimiento, desarrollo y movilidad social de un país. Fueron ejercicios de libertad y de compromiso social para transformar los contextos históricos y culturales. Las universidades latinoamericanas encontraron en la autonomía una categoría histórica que las redefinió como productoras de conocimiento y actores sociales de la educación. ${ }^{11}$

En la Universidad Nacional de México, en 1929, y a raíz de un problema estudiantil de menor importancia, pero instalado en un contexto de tensiones políticas con motivo de la sucesión presidencial, estalló un movimiento de inconformidad que rápidamente alcanzó las calles. 
La inquietud juvenil por los problemas nacionales y su deseo de libertad y autodeterminación en la educación derivó en 68 días de enfrentamientos violentos en distintas instituciones educativas de la Ciudad de México.

Para impedir que este movimiento alcanzara mayores dimensiones, el gobierno mexicano decretó la autonomía de la universidad el 10 de julio de ese mismo año. La Universidad Nacional de México es Autónoma desde 1929 y orgullosa heredera del movimiento de Córdoba de 1918.

Dos décadas más tarde, las luchas por la autonomía universitaria encontraron un espacio común en el Primer Congreso de Universidades Latinoamericanas, que aconteció en la Universidad de San Carlos de Guatemala en septiembre de 1949. En su discurso inaugural, el doctor Carlos Martínez Durán, rector de esa universidad, trazó la intención de la reunión:

Sea este Congreso [...] epopeya para conocer integralmente la misión de nuestras instituciones de cultura superior, ofreciendo soluciones para que se cumplan fielmente sus postulados, investigando la realidad americana y contribuyendo a la felicidad de los pueblos y al mantenimiento de la paz y de la libertad. ${ }^{12}$

Ese llamado tenía implícita una intención concreta. En Guatemala tuvo lugar un momento paradigmático en la historia del movimiento universitario latinoamericano. Allí se trazó un plan a seguir, pero, como bien estableció en su discurso de clausura José Roliz Bennett, decano de la Facultad de Humanidades de la Universidad de San Carlos, también se dejó en claro que la defensa de la autonomía sólo se lograría mediante hechos concretos que materializaran las intenciones de las palabras:

Compartimos todos, seguramente, la legítima tranquilidad que no ha sido éste un cónclave protocolario, erizado de formalismos, sino la reunión de un grupo numeroso de gente [...] que se ha congregado ante la urgencia de precisar un código de principios orientadores para las Universidades latinoamericanas [...]. Pero pesa también en el ambiente la preocupación de que [...] vivimos aún bajo la tradición letal de una hermandad de palabras que todos deseamos transformar en una fraternidad de hechos. ${ }^{13}$

En concordancia con esa idea, el 22 de septiembre de 1949 se constituyó formalmente la Unión de Universidades de América Latina y el Caribe (UDUAL), donde se cristalizó la lucha latinoamericana en favor de la autonomía universitaria.

\section{De "Justo Sierra 16" a la Ciudad Universitaria}

El inicio de la UDUAL fue motivo de gran celebración. El júbilo, sin embargo, duró poco, ya que al poco tiempo de ser creada la naciente organización tuvo que ajustarse a un nuevo escenario geopolítico internacional en el que dos superpotencias propusieron dos visiones opuestas de la realidad. La llamada Guerra Fría se peleó en las periferias de los dos polos antagónicos. América Latina fue un escenario muy activo de la disputa y, por consi- 
guiente, la UDUAL tuvo que erigirse en defensa de las universidades como espacios ajenos a cualquier pretensión imperial.

En octubre de 1954, Juan Gómez Millas, rector de la Universidad de Chile, y Guillermo Coto Conde, secretario general de la UDUAL, hicieron del conocimiento del entonces rector de la Universidad Nacional Autónoma de México, el ingeniero Nabor Carrillo, que el nuevo gobierno de Guatemala, emanado de un golpe de Estado auspiciado por la Agencia Central de Inteligencia de los Estados Unidos (CıA, por sus siglas en inglés), había retirado el 13 de septiembre la subvención de diez mil dólares que permitían el funcionamiento de la UDUAL desde su fundación. De esa manera solicitaron la posibilidad de que la UNAM acogiera a la aún muy joven organización:

Como la medida tomada entraña un acto inamistoso de parte del nuevo gobierno de Guatemala hacia la Unión de Universidades Latinoamericanas, que hace imposible el desarrollo normal de sus actividades en Guatemala, nos permitimos consultar a usted sobre la conveniencia de un cambio inmediato de la sede de la organización, que ha venido funcionando en la capital del país centroamericano desde su fundación. ${ }^{14}$

Consciente de la importancia del acontecimiento, el rector Carrillo dio su visto bueno a la solicitud por lo que, a inicios de 1955, las oficinas centrales de la UDUAL se localizaron provisionalmente en un antiguo edificio de la UNAM, el Colegio de San Ildefonso, ubicado en el número 16 de la calle Justo Sierra del Centro Histórico de la Ciudad de México.

Poco después, el 23 de marzo de ese año, el secretario de la organización latinoamericana se dirigió nuevamente al rector Carrillo para pedir el espacio ocupado por las oficinas del Consejo Universitario y del Patronato Universitario en el octavo piso de la Torre de Rectoría. Agregó también una solicitud de mobiliario y personal adecuados, para tener unas instalaciones "sin lujo pero decorosas" y, durante el verano, gestionó un presupuesto suficiente para cumplir con las funciones de la organización.

Consciente de la importancia de asegurar la supervivencia y desarrollo de una institución que guardaba en sí la esencia de la autonomía universitaria en el continente, las autoridades de la UNAM, encabezadas por el rector Carrillo, no dudaron en cumplir todas las solicitudes. Así, en noviembre de 1955, se estrenaron las oficinas de la UDUAL en el octavo piso de la torre más alta de Ciudad Universitaria. En la revista Universidades se hizo patente la buena la noticia:

El nuevo local de la Unión reúne condiciones óptimas, tanto por su amplitud, como por su ubicación en el corazón mismo de la Ciudad Universitaria y por las incomparables perspectivas que pueden apreciarse desde los ventanales que lo circundan. Es esta una nueva manifestación del generoso espíritu de las autoridades universitarias mexicanas para con la Unión de Universidades Latinoamericanas, que compromete la gratitud de la organización y de todos los centros universitarios asociados. 
Gracias a la solidaridad y hospitalidad de la UNAM, la UDUAL dejó pronto su condición de asilo para establecerse en su nueva casa y seguir funcionado con nuevos recursos y capacidades. En septiembre de 1959, en el marco de su III Asamblea General, llevada a cabo en Buenos Aires, se firmó la Carta de Universidades Latinoamericanas en la que se establecieron los objetivos puntuales de la organización.

En octubre de 1967, durante la V Asamblea General que tuvo lugar en Lima, la organización aprobó un nuevo estatutario donde quedó establecida la Ciudad de México como su sede permanente.

\section{La UDUAL y su defensa de la autonomía durante la Guerra Fría}

La UDUAL sólo puede explicarse si se entiende cabalmente la magnitud de la censura y la falta de libertades durante la segunda mitad del siglo XX, caracterizada por el intervencionismo de los protagonistas de la Guerra Fría. Entonces como ahora, silenciar la pluralidad y limitar las libertades significaba, por definición, asumir a las universidades autónomas como instituciones tolerantes -incluso promotoras- de la disidencia, fuera del control del Estado y, en ocasiones, acusadas de favorecer intereses extranjeros mediante la difusión de ideologías contrarias a los intereses nacionales.

El carácter universal del conocimiento, desarrollado y transmitido principalmente en estas grandes instituciones sociales que abrieron, además, espacios a sectores excluidos, fueron percibidas por los gobiernos autoritarios como una amenaza al monopolio del poder. Para quienes lo concentraban, cualquier espacio que promoviera la libertad de pensamiento mediante la docencia y la investigación atentaba contra las intenciones homogeneizadoras que, apoyadas en el miedo y la represión, buscaban imponer un modelo único e incuestionable que determinara el rumbo de los distintos países.

En otras palabras, el contexto latinoamericano durante buena parte de la segunda mitad del siglo Xx estuvo marcado por el estatismo autoritario, cuyo futuro dependía de la supresión de la crítica por cualquier medio disponible. Las universidades fueron entonces un objetivo natural.

Para 1968, con los antecedentes de las dictaduras en Brasil, Colombia, Cuba, Guatemala, Honduras, Nicaragua, Paraguay, República Dominicana y Venezuela; el Consejo Ejecutivo de la UDUAL tenía muy claro el origen del descontento de los jóvenes en América Latina y así lo estableció el informe de acuerdos de su décima segunda reunión, llevada a cabo en julio de ese año, en la Ciudad de México, antes de los primeros acontecimientos relativos al conflicto estudiantil en nuestro país:

La presente agitación de la juventud universitaria de nuestra América, antiguamente criticada con dureza por otros países, es ahora reconocida como un fenómeno de carácter mundial, que viene sufriendo el impacto de la falta de un ajuste adecuado entre los ideales como modelo de la juventud y la realidad del mundo en que vivimos. ${ }^{16}$ 
Aunado a esto, para los años sesenta ya era evidente que las embestidas en contra de las universidades seguirían siendo una estrategia sistemática para impedir el surgimiento y desarrollo de focos de crítica y oposición.

La UDUAL, consciente de las dificultades políticas por las que atravesaba la región, que se estaban convirtiendo en un factor negativo para el quehacer universitario, creó en 1957 una Comisión de Defensa de la Autonomía Universitaria.

A partir de 1967, afín con el principal objetivo asentado en el Artículo $2^{\circ}$, inciso f), de los Estatutos de la Unión de Universidades de América Latina, se estipuló: "Promover el reconocimiento y respeto de la autonomía universitaria, mediante la vigencia efectuada de las libertades de cátedra y de investigación y del régimen de autogobierno". ${ }^{17}$

El caso mexicano caracterizado por un ejercicio del poder político muy distinto al de las dictaduras de Centro y Sudamérica, en el cual no fue necesaria la toma de poder de militares o afines, estaba definido por un partido hegemónico que abría pocas oportunidades de participación política y que no estaba dispuesto a entender el surgimiento generalizado de un ímpetu democratizador.

La respuesta del gobierno en 1968, sin embargo, y guardando toda proporción, evidenció rasgos autoritarios similares a los vistos al sur de sus fronteras: represión violenta, arrestos masivos, desaparición y tortura de estudiantes y líderes sociales y toma de instalaciones de distintas instituciones de educación superior, incluidas la Universidad Nacional y el Instituto Politécnico, las cuales concentraban al mayor número de estudiantes del país, muchos de ellos provenientes de sectores poco favorecidos.

La represión y los niveles de violencia fueron la norma en las dictaduras militares latinoamericanas. En el caso de Uruguay, por ejemplo, los ataques a las universidades fueron menos velados. En este país una de las primeras medidas después del golpe de Estado fue el arresto del rector de la Universidad de la República, el doctor Lichtensztejn, que provocó una reacción de condena casi inmediata por parte de la UDUAL que, sabedora de la posición de vulnerabilidad en la que se encontraban las universidades, procuró dar a conocer toda la información disponible y rechazar enfáticamente la violación de los derechos de los universitarios y los ataques a la autonomía.

La repetición de violaciones de la autonomía en la región era tan frecuente que prácticamente en todos los informes del secretario general sobre las reuniones del Consejo Ejecutivo y de las Asambleas Generales, de 1967 a 1973, se deja constancia explícita de la preocupación que esto supone para la Unión.

En estos informes se describen los ataques a las universidades en forma de arrestos, intervenciones de fuerzas de seguridad, las violaciones a las garantías individuales, asesinatos de estudiantes, por mencionar algunas. Sin embargo, aun cuando los medios para responder a estas agresiones eran muy precarios y limitados, la UDUAL insistió en enviar comunicaciones de rechazo a los distintos gobiernos, así como utilizar la revista Universidades como un medio de información que permitiera a la comunidad académica latinoamericana conocer -y condenar- lo que iba aconteciendo. Más aún, también utilizó la red de la organización para solicitar asilo para los miem- 
bros afectados de las comunidades universitarias. En varios casos, por ejemplo, ayudó a colocar en universidades e instituciones de otros países a docentes, alumnos y autoridades desplazadas por los conflictos.

De esta forma, la UDUAL, se posicionó como una institución que, aprovechando su carácter internacional, podía defender las virtudes de la autonomía sin estar sujeta a intereses políticos particulares. Si bien los escritos de condena y la publicación de información relativa a la violación de derechos pueden ser vistos como una respuesta insuficiente, la labor y esfuerzo de esta organización impidió que el desarrollo del autoritarismo fuera visto como un problema aislado.

\section{La autonomía latinoamericana tras el Consenso de Washington: la educación como bien público y social}

De los años cincuenta a los ochenta la autonomía universitaria y la existencia misma de instituciones abiertas, libres y críticas, estuvo en constante riesgo en nuestra región. Esto explicó el desarrollo de la UDUAL, que se convirtió en una institución protagónica en la defensa de las libertades más básicas como la libertad de expresión, de cátedra y la necesidad de que las universidades pudieran gobernarse libremente a sí mismas.

Como dice la Declaración de Quito, emitida en julio de 1991, "la defensa de la autonomía universitaria hizo posible el desarrollo de la ciencia y la cultura general, además de consolidar la instauración de la educación superior en América Latina”.

Para principios de los años noventa, sin embargo, la UDUAL tuvo que replantear algunos de sus objetivos y, sin olvidar su responsabilidad de proteger la autonomía universitaria, concentrarse en desafíos colosales como revertir el rezago educativo.

La Declaración de Quito es quizás el documento que plasmó con mayor claridad el momento que la Unión de Universidades estaba viviendo. Era imprescindible aprovechar el impulso que generó la defensa de la autonomía para detectar los retos por venir e identificar las herramientas disponibles para lograr una excelencia académica que incrementara el desarrollo de la región.

A partir de este momento, el uso y desarrollo de la ciencia y la tecnología se volvió una prioridad para la UDUAL porque, con mayor conocimiento científico y tecnológico, las universidades podrían tener un mayor impacto en sus sociedades, podrían también redefinir su relación con los sectores público y privado, y reclamar una relevancia estratégica que tienen hasta nuestros días, con la convicción de que sin educación de calidad se compromete el desarrollo y la estabilidad de las democracias.

Finalmente este momento definió la declaración de la Conferencia Regional de Educación Superior, CRES 2018, que al ratificar el estatus de la educación como un bien público social y estratégico, establece como indispensable el fortalecimiento de los lazos de respeto y cooperación entre instituciones educativas libres.

A lo largo de estos años la Unión de Universidades de América Latina y el Caribe ha demostrado que las visiones unívocas del mundo, cuando se 
concentran y protegen desde el poder, no sólo niegan la libertad, sino que impiden el desarrollo y la justa distribución de los beneficios del progreso.

Habrá sin duda nuevas formas de amenazar la autonomía de las universidades y la libertad de pensamiento en nuestros territorios. El resurgimiento de la intolerancia y los falsos nacionalismos nos obliga a mantener y fortalecer nuestros lazos en una relación cuya fortaleza surge del respeto a la diversidad que complementa a la certeza compartida de que en la educación está el origen del reconocimiento, de la igualdad, de la responsabilidad social y del progreso. 


\section{Notas}

1. Gustavo García de Paredes, "En defensa de la autonomía universitaria”. Universidades, núm. 36, enero-abril 2008: 47.

2. De acuerdo con Carlos Tünnermann Bernheim, "la universidad colonial hispánica fue señorial y clasista, como la sociedad a la cual servía y de la que era expresión. Creada dentro del contexto de la política cultural trazada por el imperio español, tuvo como misión atender los intereses de la Corona, la Iglesia y las clases superiores de la sociedad”. Así, tras el desarrollo del Estado nación las comunidades universitarias latinoamericanas buscaron primero la autonomía frente al poder religioso ("La autonomía universitaria en el contexto actual Universidades". Universidades, núm. 36, enero-abril 2008: 21-23).

3. Sobre todo de profesionistas liberales: juristas y médicos, y, en menor medida, ingenieros y arquitectos.

4. De 1870 a 1914 la población de Argentina pasó de 1 millón 800 mil habitantes a 7 millones 800 mil; el comercio exterior de 74 millones de pesos oro a 1,000 millones; la red ferroviaria de poco más de 700 kilómetros a más de 33,000; y el área sembrada de 200 mil hectáreas a 12 millones 400 mil hectáreas. En pocas palabras, la población creció cuatro veces, el comercio exterior 12, la red férrea 40, y el área sembrada 55 (Ezequiel Gallo, "Liberalismo y crecimiento económico y social: Argentina (1880-1910)”. Revista de Instituciones, Ideas y Mercados, núm. 49, octubre 2008: 234).

5. Renate Marsiske, "Clases Medias, universidades y movimientos estudiantiles en América Latina”, en Renate Marsiske (coord.), Movimientos estudiantiles en la historia de América Latina, t. 1(México, Centro de Estudios sobre la Universidad/Plaza y Valdés, 2002), 144.

6. Ibíd., 145-151.

7. Ibíd., 145-146.

8. Manifiesto Liminar de Ia Reforma Universitaria deI 21 de junio de 1918.

9. Gustavo García de Paredes, "En defensa de la autonomía universitaria”, 48.

10. Diego Tatián y Guillermo Vázquez, "La autonomía hacia el centenario de la Reforma Universitaria” (Resumen Ejecutivo): Cuadernos de Universidades (México: Udual, 2018).

11. Antonio Ibarra Romero, "La autonomía universitaria: experiencias, contenidos, significaciones”. Universidades, núm. 66, octubre-diciembre 2015: 2.

12. Carlos Martínez Durán, "Discurso de bienvenida para las delegaciones al Primer Congreso de Universidades Latino-Americanas" (Archivo Histórico de la UDUAL, 1949), fojas 9.

13. Analhi Aguirre, "Palabras, hechos y diálogo, 65 años después. Comentarios sobre algunos discursos del Primer Congreso de Universidades Latinoamericanas”. Universidades, núm. 60, abril-junio 2014: 65 .

14. Archivo de la Unión de Universidades de América Latina y el Caribe, 1949-2017

15. UdUal, "Noticias de la Unión de Universidades Latinoamericanas". Universidades, 6, núm. 15, 1955.

16. Efrén del Pozo (Ed.), Historia de la Unión de Universidades de América Latina (México: UDUAL, 1976), 190.

17. Analhi Aguirre y Antonio Ibarra, "Documentos sobre la defensa de la autonomía universitaria en América Latina a través del archivo de la UDUAL", Documentos sobre la defensa de la autonomía universitaria en América Latina, a través del archivo de la UDUAL, 2014, <http://65aniversario.udual.org/index2.html>. 